\title{
PNEUMONIA AMONG AFRICANS IN UGANDA
}

\author{
BY \\ H. M. LEATHER \\ From the Department of Medicine, Makerere College, Kampala, Uganda
}

(RECEIVED FOR PUBLICATION MARCH 2, 1958)

Pneumonia is common among East African natives and in the past has been a frequent cause of death. Trowell (1932) recorded a mortality rate of $25 \%$ among 194 cases studied, while Davies (1947) found 472 deaths from pneumonia in 2,994 necropsies. The object of the present study has been to determine as far as possible the aetiology and clinical course of this illness.

Kampala, where this study was made, is situated a few miles from the northern shore of Lake Victoria at an altitude of $3,800 \mathrm{ft}$. $(1,160 \mathrm{~m}$.). The mean temperature is $71^{\circ} \mathrm{F}$. $\left(22^{\circ} \mathrm{C}\right.$. $)$ and the thermometer rarely drops below $66^{\circ} \mathrm{F} .\left(19^{\circ} \mathrm{C}\right.$. $)$ or rises above $82^{\circ} \mathrm{F}$. $\left(28^{\circ} \mathrm{C}\right.$.). The humidity is high, reaching $100 \%$ during most nights in the year and rarely dropping below $50 \%$ during the day-time. Being only 20 miles from the Equator, Kampala has little seasonal change. Coryza and pneumonia are prevalent throughout the year. Chronic bronchitis is rare, and acute bronchitis, when seen, is often part of the clinical picture of typhoid or typhus.

\section{MATERIAL}

Between September, 1955, and May 31, 1956, all patients admitted on certain routine admission days to one of the medical units at Mulago Hospital with a diagnosis of pneumonia were studied. Shortage of beds did not permit the inclusion of patients admitted with pneumonia on all admission days, but the method adopted ensured that the patients were unselected. On these study days 68 patients were admitted with a diagnosis of pneumonia, of whom 16 were found on examination to be suffering from conditions other than pneumonia. These included other acute chest diseases, pulmonary tuberculosis, and typhoid and typhus fevers. In the remaining cases the diagnosis of pneumonia was confirmed, and it is these 52 patients who were specially studied. There were 44 men and eight women. Ages ranged from 10 to 65 years. Age and sex distribution is shown in Table $I$.

\footnotetext{
*Present address: Department of Medicine, University of Bristol.
}

TABLE I

AGE AND SEX DISTRIBUTION OF 52 PATIENTS WITH PNEUMONIA

\begin{tabular}{c|c|c|c}
\hline $\begin{array}{c}\text { Age-group } \\
\text { (Years) }\end{array}$ & Male & Female & Total \\
\hline $10-20$ & 11 & 1 & 12 \\
$20-30$ & 14 & 0 & 14 \\
$30-40$ & 11 & 3 & 14 \\
$40-50$ & 7 & 3 & 10 \\
$50-60$ & 0 & 1 & 1 \\
$60-70$ & 1 & 0 & 1 \\
\hline
\end{tabular}

\section{METHOD}

A history was taken and a clinical examination made. A specimen of sputum, and, in almost alt cases, a high laryngeal swab, was taken, and bot were cultured aerobically on blood agar before chemotherapy was begun. Haemoglobin and total and differential white blood counts were estimated, and thick and thin films examined for malarial parasites. Chest radiographs were taken as soon as possible after admission. Clinical and radiological progress was studied frequently during the patient's stay in hospital and, when possible, after discharge home.

Virus studies were carried out at the Central Public Health Laboratory, Colindale, on the last 17 cases in the series. Specimens of serum were taken shortly after admission and approximately 10-14 and 14-21 days later. They were examined for immune bodies to influenza viruses A, B, and $C$, the adenoviruses, the rickettsia of $Q$ fever, and the psittacosis-lymphogranuloma venereum group of viruses, and for streptococcus M.G. agglutination. These 17 patients were Frei tested, and 0 blood from each was taken for culture before chemotherapy was begun.

Patients were treated with soluble or procaine penicillin initially. In a small number, further treatment, usually with a broad spectrum antibiotic, was necessary.

In all patients an anatomical and aetiological diagnosis was made (Fig. 1). 


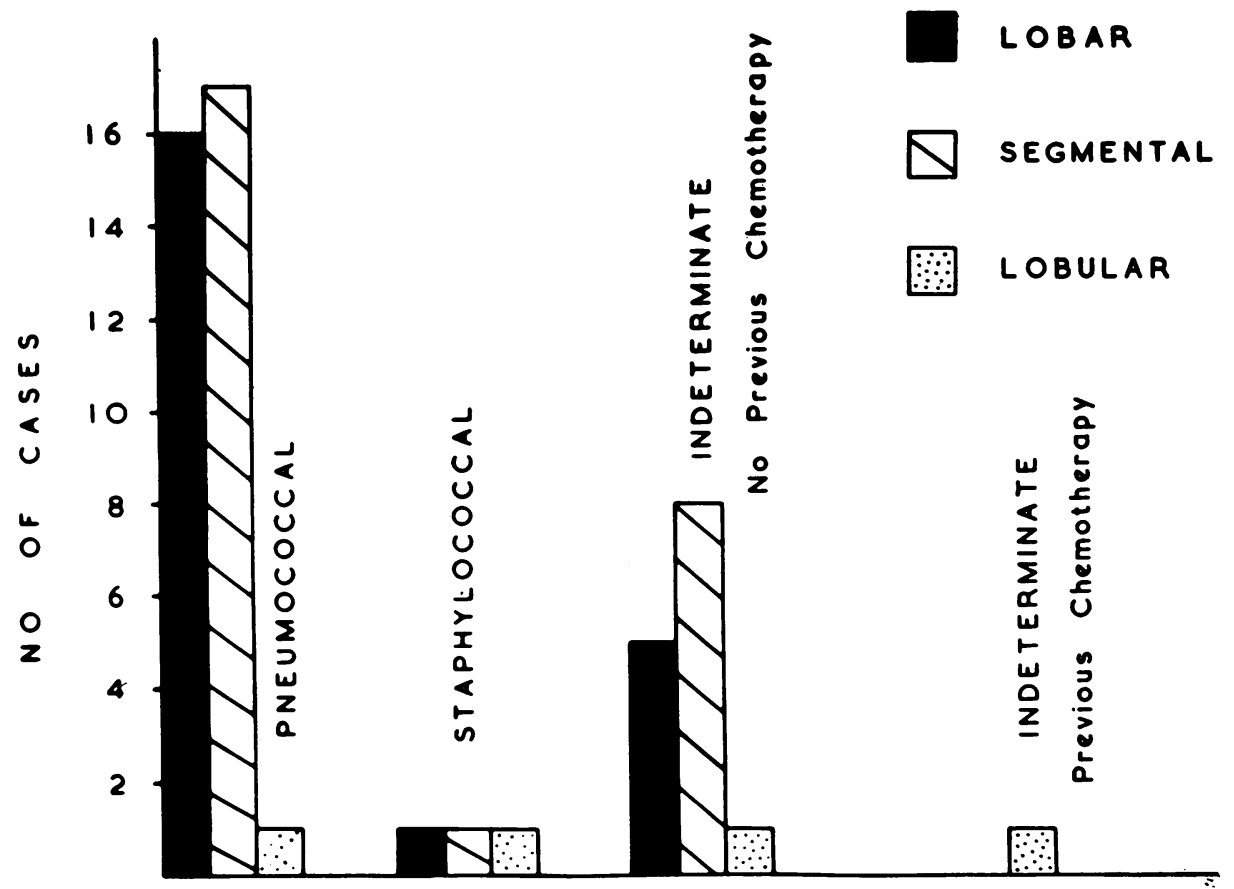

FIG. 1.-Anatomical distribution of pneumonia in various aetiological groups.

\section{Anatomical Diagnosis}

The anatomical diagnosis was made from combined clinical and radiological findings. Nearly always the one corroborated the other, for the physical signs were generally florid. Bronchial breathing was present in 46 of the 52 cases. For exact location of the anatomical lesion, however, the chest radiographs were used except in two patients where the initial radiograph had been omitted. In two cases the area of consolidation extended after the first radiographs were taken. In all the others the extent of the lesion was maximal in the first films, and it was from these that the anatomical diagnosis was usually made.

The cases were divided into four anatomical categories: Lobar pneumonia, lobar pneumonia with segmental affection of other lobes, segmental pneumonia, and lobular pneumonia. The definitions of these categories by Crofton, Fawcett, James, Scadding, Macrae, and Marmion (1951) were adopted. Thus, lobar pneumonia was diagnosed when the pneumonic process involved the whole of one lobe. Segmental pneumonia was diagnosed when a more or less uniform opacity occupied less than the whole of a lobe. In these cases the lateral radiograph usually revealed the anatomical segment or segments involved. Pneu- monia was considered to be lobular when the pneumonic process produced a patchy opacity radiologically in one or both lung fields.

There were 26 patients with segmental pneumonia. In 20 only one lobe was involved; in five cases two lobes were affected, while in the remaining patient the pneumonic process had invaded segments in four lobes. Even in the 20 cases where a single lobe only was affected, 14 had involvement of more than one segment. The distribution of lesions in the $\mathbf{3 4}$ lobes affected in these 26 patients is shown in Table II. In upper

TABLE II

DISTRIBUTION OF ANATOMICAL LESIONS IN 52 CASES OF PNEUMONIA

\begin{tabular}{r|c|c|c|c|c}
\hline & R.U.L. & L.U.L. & R.M.L. & R.L.L. & L.L.L. \\
\hline $\begin{array}{c}\text { Group I: Segmental pneu- } \\
\text { monia (26 cases) }\end{array}$ & 6 & $8^{*}$ & 5 & 6 & 9 \\
$\begin{array}{c}\text { Group II: Lobar pneu- } \\
\text { monia (12 cases) }\end{array}$ & 2 & 1 & 3 & 6 & 5 \\
$\begin{array}{c}\text { Group III: Lobar pneu- } \\
\text { monia with segmental } \\
\text { change: (10 cases) }\end{array}$ & & & & & \\
$\begin{array}{c}\text { Lobar component .. } \\
\text { Sogmental component }\end{array}$ & 1 & 0 & 3 & 1 & 6 \\
$\begin{array}{c}\text { Group IV: Lobular pneu- } \\
\text { monia (4 cases) }\end{array}$ & 1 & 0 & 0 & 3 & 3 \\
\hline Total .. & 17 & 12 & 12 & 18 & 23 \\
\hline
\end{tabular}

* Includes four cases with involvement of lingula. 
lobe lesions anterior and posterior segments were equally involved. The apical segment was, however, affected in only one patient.

Twenty-two patients had lobar pneumonia. In 17 one lobe and in four two lobes were involved. In one patient the whole right lung became pneumonic ; only the right middle lobe was affected on admission, but the disease process spread to the rest of the lung during the first 48 hours in hospital. In 10 of the patients with lobar pneumonia segmental lesions were present elsewhere, usually in the upper lobes.

The average age of the group with segmental pneumonia was 34 years, and of the lobar group 29 years. A slightly higher proportion of patients
Lobular pneumonia was diagnosed four times. All the patients were between 40 and 50 years of age. In two of them small areas of lobular pneumonia affected basal lower-lobe segments associated with bronchiectasis, and no pathogenic organisms were isolated from the sputum or laryngeal swab. The third patient had lobular involvement of the left lower lobe and anterior segment of the left upper lobe. The history was short, pneumococci were isolated from the sputum, the white blood count was high, and a good response to penicillin was obtained. In the fourth patient a diagnosis of staphylococcal pneumonia was made on clinical grounds. This case is discussed more fully below.

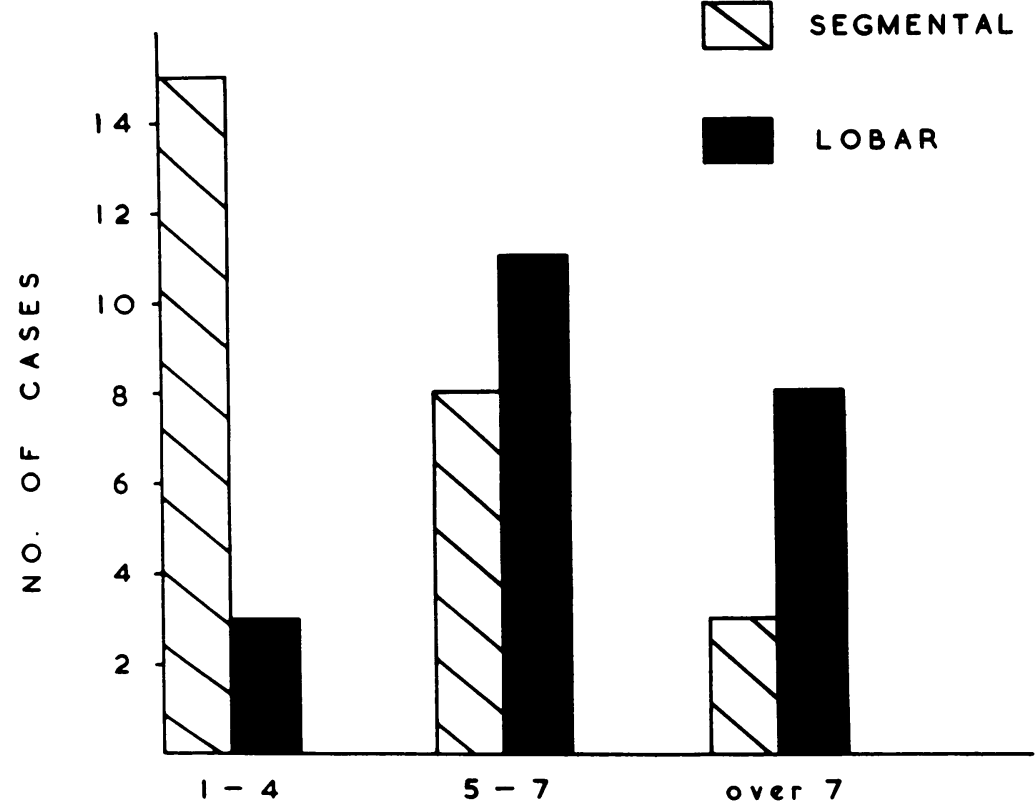

DAYS OF ILLNESS PRIOR TO ADMISSION.

FIG. 2.-Duration of illness before admission in relation to anatomical distribution of pneumonia.

with segmental pneumonia gave negative bacteriological results than of those with lobar pneumonia. In general, however, the onset of the illness, clinical picture, naked-eye appearance of the sputum, and leucocyte response was the same in both groups. The only notable difference between these two anatomical groups, lobar and segmental, lay in the duration of illness before admission to hospital (known in 47 patients), which tended to be shorter in the segmental group than in the lobar (Fig. 2).

\section{Aetiological Diagnosis}

Aetiologically the cases fell into two groupsthose of bacterial pneumonia and those of pneumonia of indefinite aetiology. The diagnosis of bacterial pneumonia depended on the isolation from the sputum, laryngeal swab, blood, or lung after death of the pathogenic organism and the presence of a compatible clinical picture. The group of pneumonias of indefinite aetiology in cluded all those patients with pneumonia in whom no pathogenic organism was isolated. 
Pneumococcal Pneumonia.-There were 34 cases of pneumococcal pneumonia. The organism was isolated from both the sputum and the laryngeal swab in 22 of the 25 cases in which both were cultured. In nine of the others the organism was isolated from the sputum and in the remaining three from the laryngeal swab, no sputum having been obtained. Blood culture was positive in only two of the 17 cases in which it was carried out.

In 17 cases the lesion was segmental; in 16 lobar, including eight with segmental change in other lobes; and in the remaining one it was lobular.

The onset was generally sudden with shivering, pleuritic pain, and cough. Rusty sputum was frequent. Half the patients had had a preceding coryza and a third had been soaked by rain during the week before the illness began. Physical signs of consolidation were generally well developed and bronchial breathing was present in the entire lobar group and in all but four with segmental pneumonia. Nearly all patients had a good leucocyte response, amounting to more than 15,000 polymorphs per c.mm. in over half and to more than 20,000 per c.mm. in 12 . Only three of the 34 patients, all with segmental pneumonia, had less than 6,000 polymorphs per c.mm., and of these two had been ill for more than two weeks before admission. Malarial parasitaemia was never seen.

Virus studies were made in 10 of the 34 patients with pneumococcal pneumonia. One showed evidence of recent infection with influenza $A$. He made a complete recovery from a moderately severe segmental pneumonia associated with a good leucocytosis. Pneumococci had been isolated from both sputum and laryngeal swab. The remaining nine cases all gave negative results except for raised titres to the psittacosis-lymphogranuloma venereum group of viruses in three cases. The titres, however, remained stationary throughout the illness in these three cases and the Frei test was strongly positive in all. These illnesses were in every other respect characteristic of pneumococcal pneumonia, and it was concluded that the raised titres in these patients were not connected with the present condition but with past lymphogranuloma venereum infection, which is not uncommon in Uganda.

Staphylococcal Pneumonia. - There were three patients in whom a diagnosis of staphylococcal pneumonia was entertained, two of whom died. Both the patients who died were middleaged men who were very ill on admission. One died shortly afterwards and before any investigations had been made. At necropsy the whole left lower lobe was found to be pneumonic, and from a swab taken from the lung at necropsy pneumococci and Staphylococcus aureus were cultured.

The other death occurred in a known chronic alcoholic who had been ill for five days with fever, cough, pleuritic pain, and who was stuporose on admission. Scattered areas of consolidation in both lungs led to a clinical diagnosis of lobular pneumonia. The sputum was rusty and the temperature $101.2^{\circ}$ F. $\left(38.4^{\circ}\right.$ C. $)$ The leucocyte count was 11,550 per c.mm. with $88 \%$ polymorphs, and sputum culture yielded Staphylococcus aureus. The patient made no response to chemotherapy and died within 24 hours of admission. Necropsy was refused.

The third case was in a young man suffering from multisegmental pneumonia of the right lower lobe. A light growth of Staphylococcus aureus was obtained from both sputum and laryngeal swab. The patient responded well to treatment with penicillin, the chest radiograph virtually clearing within 21 days. In this patient there was a high titre of antibodies to the psittacosis-lymphogranuloma venereum group without further rise, and the Frei test was strongly positive.

Other Bacterial Pneumonias.- No case of Friedländer's bacillus pneumonia was diagnosed in this series, although in two cases Friedländer's bacillus was present in moderate growth in a mixed bacterial flora. In neither case was the clinical picture characteristic of that associated with Friedländer's pneumonia, and as the organism was not predominant in the sputum the pneumonia in these patients has not been classified as being due to this bacillus.

Tuberculous Pneumonia.-During the course of this investigation four patients were admitted to the ward with physical signs of consolidation but were excluded from the series, as pulmonary tuberculosis was readily diagnosed from the history and general clinical examination, and confirmed by the finding of acid-fast bacilli in the sputum.

All sputum examinations in this study included a search for acid-fast bacilli, and where tuberculosis was suspected several specimens were examined. No case of coincidental infection was found. Four of the 52 cases showed radiographic evidence of old, healed, primary tuberculosis.

Virus Pneumonia.-Apart from one case with a recent infection with influenza $A$ no evidence of virus pneumonia was found in the 17 consecu- 
tive cases in which studies were made. Those cases with raised psittacosis-lymphogranuloma venereum titre and positive Frei test have already been mentioned. No case of $Q$ fever was encountered, and this condition has not so far been described in Uganda, though it has been found in Kenya (Craddock and Gear, 1955).

INDETERMINATE GROUP.-Fifteen patients fell into this category, of whom only one had had previous chemotherapy. Five had pneumonia of lobar, eight of segmental, and two of lobular distribution. Of the five patients with lobar consolidation, one died shortly after admission from a very severe right lower lobe pneumonia and no bacteriological specimens were obtained. Two had a short history, with labial herpes in one ; both had a very good leucocytosis and in both the pneumonia responded quickly to penicillin. It was thought that all three patients had bacterial pneumonia in which the causative organism had not been isolated.

Six of the eight patients with segmental pneumonia of indeterminate aetiology gave a short history and in five the illness started with coryza ; four of them had been wet through by rain during the week before the illness began. All were very ill on admission to hospital. One had labial herpes and was jaundiced. In five of these six patients the leucocytes were counted on admission and in four a moderate leucocytosis was found. Sputum culture from one revealed a mixed growth of organisms in which some pneumococci were present, but no pathogens were grown from four others, one of whom had already received chemotherapy. In the remaining patient culture of the sputum was omitted. Virus studies were performed in four of the patients and were negative. All six patients made an excellent response to treatment, the temperature usually settling within 72 hours of admission. In those followed up, radiological recovery was generally complete. These six patients were also thought to have had bacterial pneumonia.

In the remaining six patients with pneumonia of indeterminate aetiology, the nature of the illness was somewhat different. In two with a lobar distribution the onset was not sudden and both had been ill for more than 14 days before admission to hospital. The leucocyte count was low in one and 24,000 per c.mm. in the other. In one the sputum was foul and yielded an almost pure growth of $B$. proteus on culture. Both patients responded slowly to treatment and resolution was incomplete. Two patients of the six in this group had segmental pneumonia and had been ill for more than three weeks before admission to hospital. In one in whom the left lower lobe was involved, the leucocyte count was normal and the response to treatment slow, though the lesion gradually cleared. In the other patient, who had a chronic productive cough, the apical segment of the right upper lobe was consolidated (the only patient in the whole series to have an isolated affection of this segment) and the lingula was also involved. Sputum was foul. Little clearing of the right upper lobe took place over the course of six months, though the inflammatory process in the lingula resolved quickly. The remaining two patients had lobular pneumonia affecting basal segments of the lower lobes and both gave a history of recurrent chest infections. They were subsequently shown by bronchography to have bronchiectasis. In one a leucocytosis was present. Both responded slowly to chemotherapy.

In these six patients the slow onset of the illness (which may have been the reason for a late admission to hospital), together with a previous history of respiratory infection, negative bacteriological findings, foul sputum, and a slow response to treatment, suggested the possibility of an "aspiration " aetiology (Ramsay and Scadding, 1939 ; Scadding, 1948, 1952).

\section{Differential Diagnosis}

The clinical features of acute pneumonia are so striking that mistakes in diagnosis should rarely occur. This general rule applies to the patients in this study, most of whom presented a textbook appearance of pneumococcal pneumonia. In nearly all the 16 admitted during the study but found to be suffering from conditions other than pneumonia, a careful history and physical examination was sufficient to reveal the correct diagnosis. Five of these 16 had typhoid and one had typhus fever. Physical signs in the chest, however, were those of acute bronchitis rather than pneumonia. Consolidation of the lung was seen only once, in a middle-aged man, who had segmental consolidation of the right middle lobe on admission; but the diagnosis of enteric fever was suggested by the clinical picture, a rising Widal titre, and the diagnosis of typhoid fever in three other members of his family. An initial response to chloramphenicol was not sustained, and the consolidation of the lung persisted. A month after admission, tubercle bacilli were found in the sputum, several previous examinations having been negative, and shortly afterwards miliary tuberculosis developed. The pulmonary consolidation was thought to be related to the tuberculosis rather than to the typhoid fever. 


\section{Prognosis}

Three patients died, all within 24 hours of admission. Two had lobar and one lobular pneumonia. All were almost moribund when first seen.

In 13 patients who left hospital after only a short stay (usually of less than a week) the final result was not known as the lung lesions were still unresolved on discharge from hospital, and they did not subsequently attend for further examination. However, all were much improved clinically when they left hospital, and the prognosis was thought to be good. Of the remaining 36 patients, 10 showed complete resolution; and in a further 17 resolution was nearly complete, the only radiographic abnormality being small areas of linear collapse or slightly deficient re-aeration in the affected parts of the lung. The duration of study of these patients varied from six days to six months from the time of admission. It is likely that many of those in whom recovery was nearly complete would become entirely normal with the passage of time. This was illustrated by one patient who at 42 days from the time of admission had still slight radiographic change, but in whom the chest radiograph was clear a month later. Nine of the 36 cases showed incomplete resolution 28 days after the date of admission, though further improvement would probably have taken place subsequently in some of them.

Apart from jaundice in several cases on admission, the incomplete resolution in these nine patients was the only complication of pneumonia seen in this series.

It was found that the patients in whom pneumonia resolved best were those admitted early in the course of their illness. Thus, in the 27 patients known to undergo complete or nearly complete recovery, the average duration of illness before admission was 5.3 days. In the nine patients still unresolved after 28 days from the time of admission, the comparable figure was 13.4 days.

Patients with segmental pneumonia had a higher incidence of complete or nearly complete resolution than patients with lobar pneumonia (Table III). Presumably this was partly because of the tendency towards a shorter duration of illness before admission (Fig. 2) and partly because the lung lesions were less extensive. Thus, of the 19 patients with segmental pneumonia who were followed up, 17 recovered completely or nearly completely, while only two showed incomplete resolution after 28 days. Of the 15 patients with lobar pneumonia who were followed up, resolution was complete or nearly complete in 10
TABLE III

RELATIONSHIP BETWEEN DEGREE OF RESOLUTION OF PNEUMONIA AND DURATION OF ILLNESS BEFORE ADMISSION AND ANATOMICAL DISTRIBUTION

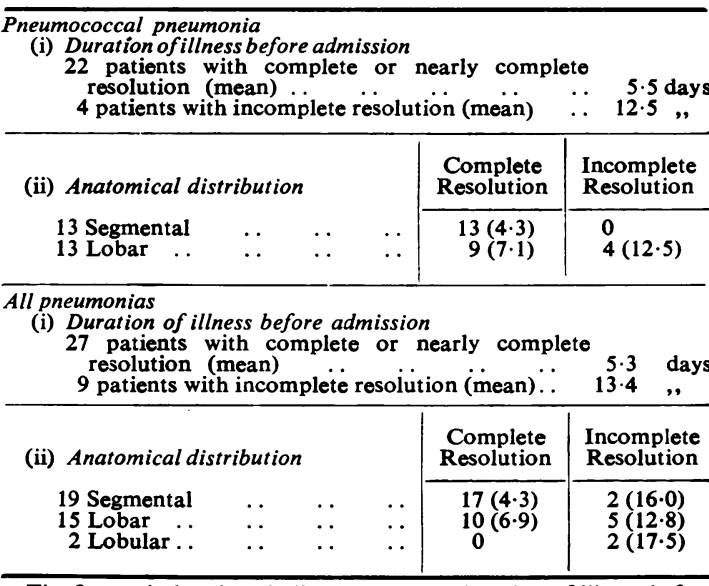

The figures in brackets indicate the mean duration of illness before admission.

(average duration of illness before admission 6.9 days) and incomplete in five (average duration before admission 12.8 days).

\section{Discussion}

Few patients in the present study were over 50 years of age. This is a reflection of the small percentage of old people in general who are admitted to hospital, and does not imply that pneumonia is uncommon in the elderly African. The high incidence in men as compared with women is in approximately the same proportion as the total figures for the year 1955, in which pneumonia was the final diagnosis in 337 men and 72 women admitted to the medical wards of the hospital. This sex difference is only partly accounted for by the different admission rate for men and women which, during the period of this study, was in the ratio of three men to one woman. No particular tribal susceptibility to pneumonia was observed.

In two-thirds of the patients the pneumonia was pneumococcal, while in another sixth the clinical picture was suggestive of pneumococcal pneumonia in every respect except that the organism was not isolated. These figures accord with the findings of Davies (1947), who analysed 472 necropsies at Mulago Hospital. Causal organisms were isolated in 170 of these, pneumococci being found in $90 \%$. The remainder were mostly due to staphylococci or Friedländer's bacillus. These findings are in contrast with those of Crofton and others (1951) from a hospital in west London, where out of a total of 110 patients with 
pneumonia they found only 29 (21\%) with a proved pneumococcal aetiology, and nearly a quarter of the patients showed serological evidence of virus or rickettsial activity. Such evidence was present in only one of the African patients here reported, but as only 17 consecutive cases were studied and cold agglutination tests were not made, more work will be necessary before the virus pattern is established. It would, however, appear from this study that among Kampala Africans pneumonia is more frequently due to bacteria and less frequently to viruses than among Londoners.

Patients with pneumococcal pneumonia nearly always had a good leucocytosis. Jacob (1954), on the other hand, found that only $61 \%$ of patients " admitted ... . with a diagnosis of lobar pneumonia" had polymorph counts above 6,000 per c.mm., and levels were in general lower than in the present series. Radiological studies were not mentioned, and it appeared that in some cases the diagnosis of pneumonia was in doubt. This led Jacob herself to comment that " it would, perhaps, be more strictly accurate to refer to the whole group as acute respiratory infection rather than lobar pneumonia." It seems likely that the discrepancy can be explained on these grounds.

According to Scadding (1952), “ Pneumococcal pneumonia in man typically assumes a lobar distribution in one or more lobes of the lung ... in a high proportion, perhaps $20 \%$ of typically ' lobar' cases, lobular foci can be found either radiologically during life, or at necropsy, in lobes other than those principally involved." Crofton and others (1951) likewise found " a clear bias towards a lobar distribution in pneumococcal pneumonia " ( 21 out of 29 cases). The most frequent distribution among the 34 cases of pneumococcal pneumonia here described was segmental. In most of the 17 patients who showed this distribution more than one segment was involved, sometimes in more than one lobe ; and in half the 16 patients who had a lobar distribution segmental consolidation was present elsewhere. Only one patient had a lobular distribution. The reason for this frequencey of segmental distribution is not clear.

One possibility is that the pneumonia in these cases arose by "aspiration," and in support of this half the patients with pneumococcal pneumonia did give a history of recent coryza. However, the clinical picture, the rapid response to treatment, and the isolation of the pneumococcus distinguished these cases from typical localized aspiration pneumonia (Scadding, 1952). Furthermore, those patients who gave a history of coryza did not necessarily develop segmental lesions. A more probable explanation lies in the duration of illness before admission. Those admitted within a few days of the onset of their illness usually had segmental pneumonia (Fig. 2); of the seven patients admitted within 24 hours of the clinical onset six had only segmental change. Those patients, on the other hand, whose illness had lasted longer than a week usually had a lobar distribution. As most of the patients in this study had been ill for less than five days this may explain why segmental pneumonia was so common.

\section{SUMMARY}

Fifty-two African patients with pneumonia have been studied. In 37 there was aetiological evidence of bacterial pneumonia, which in 34 cases was pneumococcal and in three staphylococcal. In 15 cases no organism was isolated: nine of these were thought to have had bacterial pneumonia, the remaining six were probably aspiration pneumonias. Serological virus studies were made on the last 17 patients in the series. In one there was evidence of recent influenza $A$ infection.

In pneumococcal pneumonia the commonest anatomical distribution was segmental or polysegmental. Possible reasons for this are discussed. There were three deaths, including two of the three patients with staphylococcal pneumonia. In the remainder chemotherapy usually produced a rapid response, and follow-up studies showed that in approximately four-fifths of cases the resolution was radiologically virtually complete.

The virus studies in this work were undertaken by Dr. P. S. Gardener, of the Virus Reference Laboratory, Colindale, and I also record my thanks to the Virus Research Laboratory, Entebbe, for help in preparing the sera for transport. The haematological studies were made by Mr. J. Kyobe, of the Makerere College Medical School. Dr. J. H. Middlemiss, of the Bristol Royal Infirmary, kindly assisted in the interpretation of the chest radiographs. I wish to thank Professor A. W. Williams, of Makerere College, for his encouragement and help throughout this study, and Professor C. B. Perry, of Bristol University, for his advice in the preparation of this paper.

\section{REFERENCES}

Craddock, A. L., and Gear, J. (1955). Lancet, 2, 1167

Crofton, J. W., Fawcett, J. W., James, D. G., Scadding, J. G. Macrae, A. D., and Marmion, B. P. (1951). Brit. med. J., 2, 1368. Davies, J. N. P. (1947). E. Afr. med. J., 24, 289.

Jacob, G. F. (1954). Ibid., 31, 367.

Ramsay, H., and Scadding, J. G. (1939). Quart. J. Med., 8, 79. Scadding, J. G. (1948). Lancet, 1, 89.

(1952). In Marshall, G., and Perry, K. M. A., Diseases of the Chest, Vol. 1, p. 71. Butterworth, London 